\title{
Human-Robot Scaffolding, an Architecture to Support the Learning Process
}

\begin{abstract}
Recognizing and diagnosing learner's cognitive and emotional state to intervene assertively is an important aspect to importance to improve the process. This mission that can be supported by social robots in educational contexts. A cognitive architecture to manage the robot social behavior with handling capacity is presented. The human-robot scaffolding architecture is composed of three systems: multimodal fusion, believes, and scaffolding. Those recognizes verbal and nonverbal data from user and from the mechanical assembly task, acknowledges the user's cognitive and emotional state according to the learning task and configure the actions of the robot based on the Flow Theory, which establishes relations between challenges and skills during the learning process. The theoretical analysis and explorative actions with children to build each subsystem of architecture are presented. The present research contributes to the field of human-robot interaction by suggesting an architecture that seeks the robot's proactive behavior according to learner's needs.
\end{abstract}

Keywords: Social Robots, Scaffolding, Constructionism, Human-Robot Interaction.

\section{Introduction}

During the learning process, the social robots can give physical, cognitive and emotional support based on the learner's characteristics. In the physical aspect, robots can assist the user during the mechanical assembly process by manipulating and reorganizing the blocks. For example, robots can change the blocks to foster new ways of thinking about the problem. In the cognitive aspect, robots can give support through three strategies: focus lessons, guided instructions, and collaborative work. For example, if the user is so confused then robots teach specific lessons through verbal cues or prompts. And finally, they can give emotional support to encourage the user during the learning process. For example, when the user is bored robots show a happy face to foster learners to continue the learning process. In overall, the assertive robot intervention allows to stablish, maintain, change, and finish learning events through cognitive, emotional, and physical strategies.

In the field of education, there is research interest in how to use the artificial cognitive systems to support learning processes which have been historically supported by humans. Some advancements are related to topics like: relation between physical presence and perceived support effect [6][29]; mechanisms of emotional communication [28]; non-verbal language [35] and non-verbal user behavior recognized by robots [46]. However, cognitive conditions of current anthropomorphic robots allow use them as collaborative agents in collaborative learning tasks.

\section{Architecture Development}

System I: multimodal fusion

As mentioned above, the proposed artificial cognitive architecture in three modules is presented in Figure 1. The first module acknowledges the student's behavior (multimodal fusion). The proposed method recognizes the tradition of the work developed at the Technische Universität München around the JAST project. Having said this; The architecture recognizes actions of verbal and non-verbal communication of the subject [2]. The robot interprets integrates the information of the different communication channels and estimates the cognitive state of the user [3][4]. The context for interpreting information is the mechanical task, which is related to the solution of a transformation problem. That is a problem with a defined space [30][32][33][48]. The actions developed by the robot contribute to foster the learner's cognitive development [31]. 


\section{Sensorial processing subsystem}

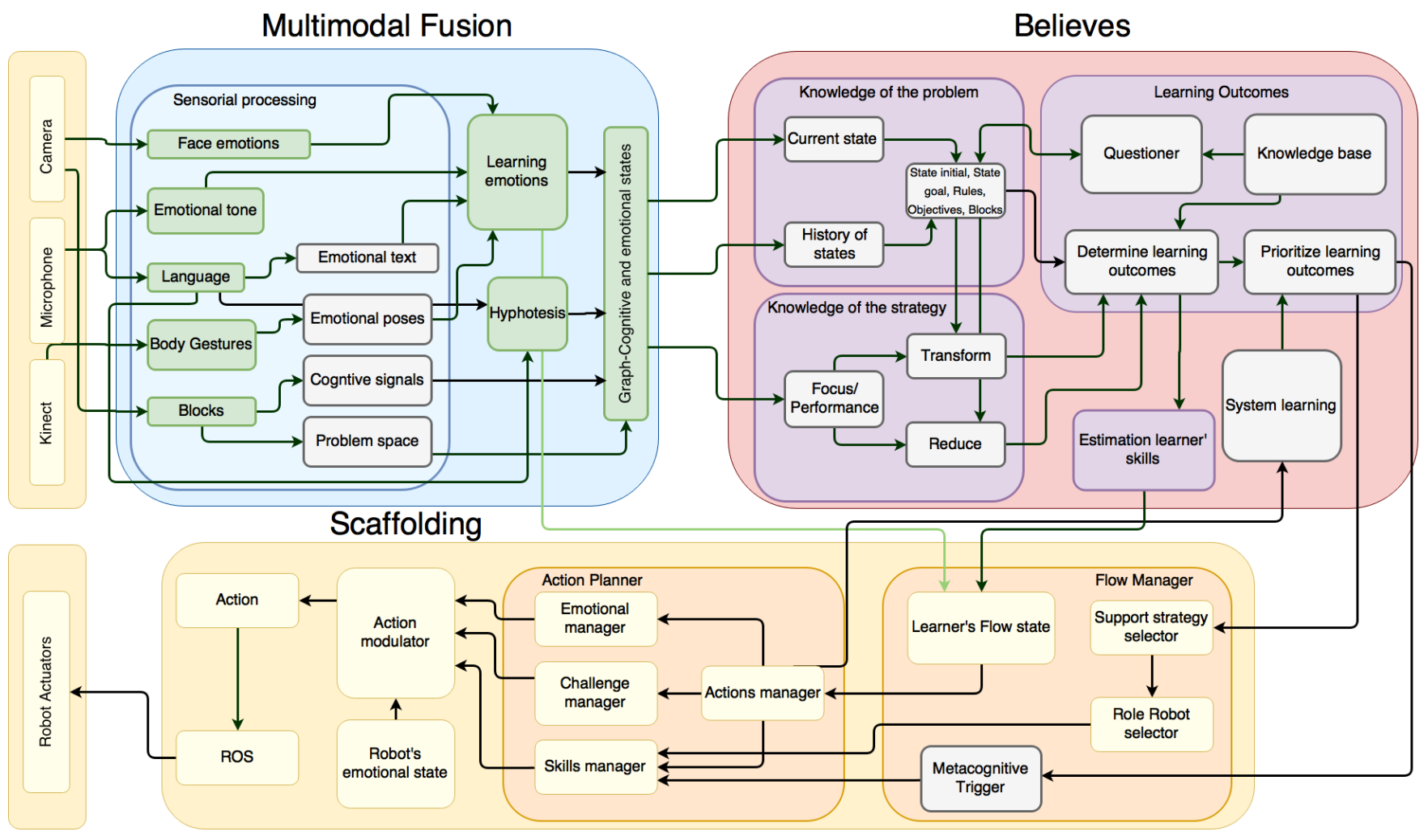

Figure 1: Human-Robot Scaffolding Architecture

The observation of the student's behavior is estimated by modules as blocks' positions, blocks' movements, emotional state, verbal judgments and cognitive gestures, as can be seen in the same figure.

The positions of the blocks allow to determine two aspects: the knowledge of the problem and the knowledge of the strategy to solve the problem. The knowledge problem is linked to the use of the operator's (an action to change a state) problem, application of rules, and the knowledge of the different states (initial, intermediate, goal) during the process to solve the problem. The knowledge to develop the strategy involves three objectives: to transform, to reduce and to apply. They are part of the Mean-End Analysis strategy. On the other hand, blocks' positions are described through three-dimensional coordinates according to mechanical restrictions. With each movement, a new node is created in the user's problem space. Some tests have been carried out with a camera and a Kinect device, although an accelerometer device into each block could be another option.

The kinematic description of the blocks is measured during each change of the state of the problem developed by the learner. This contributes to acknowledge the learner's cognitive process within the problem's solution space. For example, the variation of block's trajectory indicates changes on the user's reasoning [8], the object manipulation find out relationship between concepts and spatial thinking, and recognition of spatial patterns of the problem promotes the choice of operators even by omitting deliberative processes (National Research Council, 2005).

In order to develop the kinematic descriptions each coordinate is taken during the trajectory between two nodes is measured. Based on these data three quantitative descriptors are determined: block's velocity, change of trajectory, and blocks' dropping. Figure 2 presents the analysis of four trajectories generated by some students. In task context, the trajectories generated by the movement of the blocks could indicate cognitive actions such as hypothesis evaluation, goal change, safety and insecurity in knowledge. For example, a continuous block's trajectory means self-confidence; a change of direction or bounce indicates a change of objective. To contrast the theoretical issues, the code has been implemented using OpenCv and Python. The technological tools could be the same options which was proposed above.

The emotional state indicates the user's cognitive mood to undertake, develop and complete a learning task. The cognitive arrangement depends on two aspects: The user skills and the challenge of the problem [16]. The combination of both variables 
generates three emotional states: anxiety, flow, and boredom [21]. These can be divided into eight areas: anxiety, concern, comfort and optimism, curiosity, interest, indifference, and boredom [27].

In order to determine the user emotional learning's states different data from face movement are taken. Some alternative software as Emotient and Affective have been tested during the design of this architecture.

The verbal judgments allow to give sense to the cognitive processes and the knowledge of the user during the process of problems solution. Cognitive processes are related to aspects such as thought, attention, strategies, knowledge, and hypothesis. The interpretation of verbal judgments has two background aspects: the context of the task and the cognitive processes [12]. According to the information processing theory, the context of the task determines the operators, the rules, and the knowledge to solve the problem [37]. With this data, the emotional, cognitive and meta-cognitive states are recognized. According to the Intelligent Tutors Systems, the most use characteristics so to model to the student profile are: knowledge level $(52.8 \%)$, cognitive features $(40.75 \%)$, affective features $(16.85 \%)$, misconceptions $(15.75 \%)$, and meta-cognitive features $(6.74 \%)$ [10]. Alternatives as CMU Sphinx to recognize emotions and Synesketch to recognize textual emotion recognition have been tested to design this architecture.

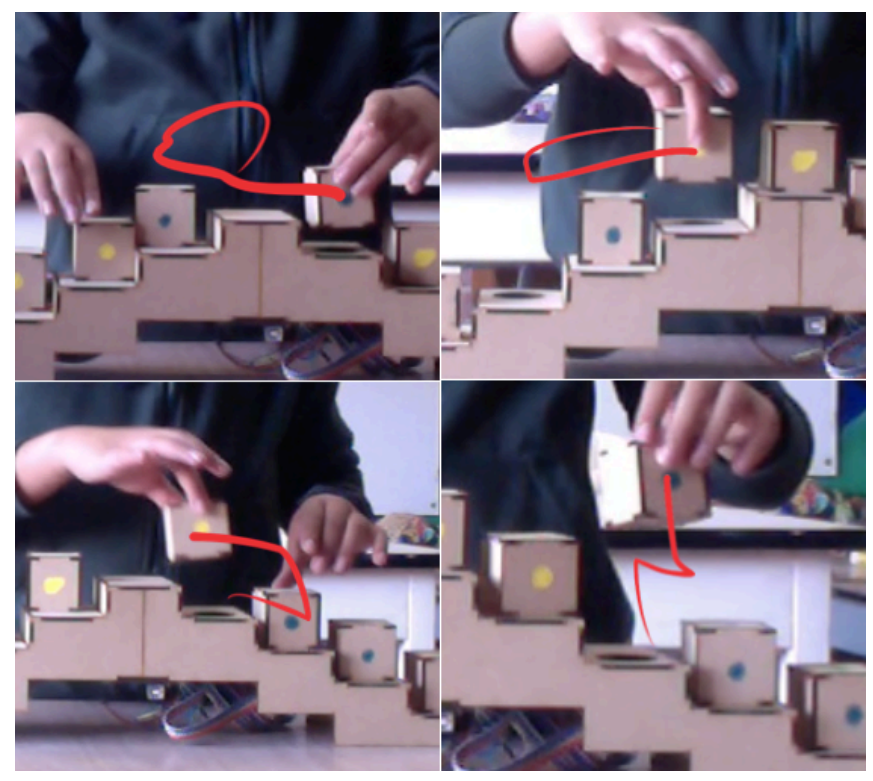

Figure 2: Kinematic characteristics

Body gestures expose the actions of thought in the problem-solving process. Characteristics such as body posture, facial expressions, eye movement and hand movement have been studied [17], based on the degree of abstraction, during the problemsolving process. The study of gestures involves three aspects: recognition, cognitive contribution, and estimation of mental models. First, the recognition involves coding gestures according to the characteristics of the problem, and segmenting them according to their occurrence, size, and quantity [36]. Second, cognitive contribution involves recognizing how its occurrence evokes implicit knowledge, promotes spatial representation, and introduces information to solve the problem [1]. Finally, but not least, is the estimation of mental models to acknowledge aspects such as knowledge of strategy, strategy change, problem difficulty and solver's expertise [11][1].

Different body movements during the problem-solving process have been analyzed. This kind of movements with or without blocks are useful to carry out assertive robot's intervention. Figure 3 presents four typical cognitive gestures presented in experimental sessions during problem-solving process: confused, spatial reasoning, iconic movements, and the intention to apply an operator and change the problem state. The optional devices to get data from fingers, wrist, arm, shoulders and head movements are cameras, Kinect, and particularly RealSense by Intel.

In general, the main methods of perception used in robots like Kismet, Cog, iCub, GRACE, Robox, Reckman, Robovie, RUBI, AMARIII, Papero, Huggable, MEXI, ROMAN, BARTHOC, BIRON, Fritz, ASIMO, iCat, AIBO, Albert Einstein and YouBot are presented. Three actions are highlighted. First, extraction of characteristics of signals like video, audio, tactile and sensors. Second, reduction of dimensionality through techniques such as analysis of main components, analysis of linear discriminants and preservation of local projections. Third, semantic comprehension through the recognition, tracking and segmentation of objects [20]. 
As a conclusion of System I, the information obtained from the sensorial processing module is organized into the graph generated by the transition of states during of solution of the transformation problem.

According to Figure 4, the nodes indicate the changes of the state in the problem space, and the arcs represent the relation between cognitive gestures, verbal judgments, and emotional states.

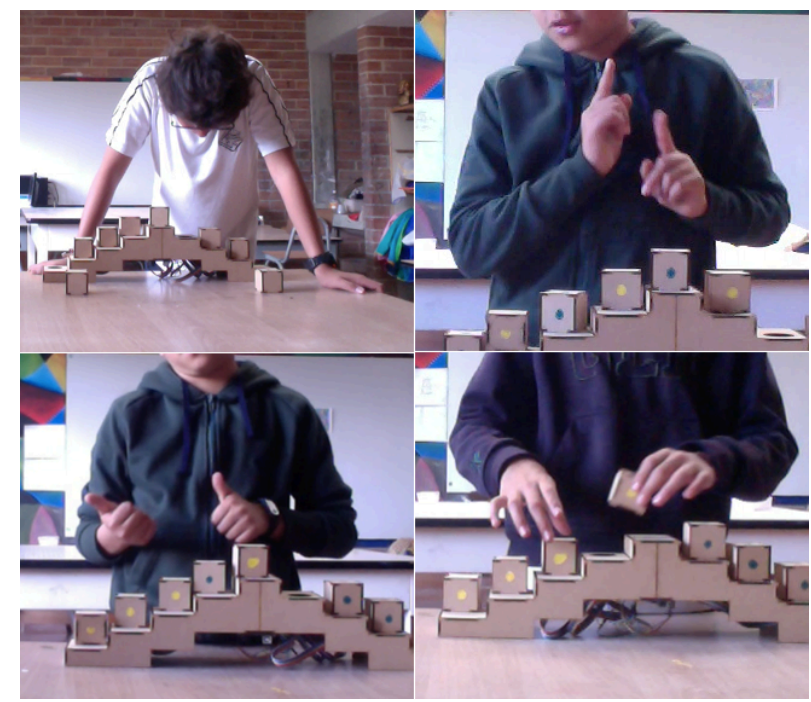

Figure 3: Cognitive gestures

\section{SYSTEM II: BELIEVES}

As mentioned above, Figure 1 shows the proposed artificial cognitive architecture in three modules. The second module diagnoses cognitive and emotional states (believes). The main objective is prioritizing the learning goals through four aspects: the knowledge of the problem, the knowledge of the strategy, the learning objectives and questioner module [10]. (See figure 1).

\section{Subsystem: knowledge of the problem}

The problem knowledge level is determined through the student's behavior observation. Characteristics as the initial state, goal state, intermediate states, operators, errors, misconceptions, and rules are analyzed. In the transformation problems as Hanoi Tower, errors are detected by infringing rules and misconceptions are detected through the development of the strategy [14].

The current state module takes information from the previously constructed graph. Figure 4 presents a bit of the graph. The module evaluates the learner's actions related to the problem. The actions describe the learner's procedural knowledge related to the use of rules and operators. The evaluation process means the user's knowledge of the problem. With this information, the objectives for procedural support are estimated.

The history states module takes information from the previously constructed graph. The module evaluates the learner's actions related to use of means-ends analysis strategy. The learner's actions describe their cognitive knowledge related to two objectives of this strategy. First, to transform (to find the difference between the current state and the target state). Second, to reduce (to find the operator that reduces the difference). As a result, the evaluation indicates the knowledge of the strategy. With the above information, the goals for cognitive support are estimated.

\section{Subsystem: knowledge of the strategy}

The strategy knowledge level is acknowledged through data of the task. The cognitive characterization process involves four steps: labeling data, distilling data features, developing detector and validating. Data as time invested in the transition of each problem state and previous interactions are evaluated [13]. Also, the metacognitive characteristics as self-regulation, selfevaluation, self-explanation, and self-efficacy could be assessed.

The memory state sequence shows aspects as the learner's thinking states and the doubts and pauses which correspond to changes in the direction of thought. Each memory state is joined to a knowledge about the solution process. This kind of information is useful to determine the cognitive student needs (transforming, reducing) which are necessary to learn the meanend analysis strategy. Also, the information of memory states is useful to estimate the learner's space problem, which is a dynamic structure and it is defined by sub-goals (transforming, reducing, applying), storage goals, connection table, structure goals, and production system. 
Different methods of cognitive task analysis as think-aloud protocols, content analysis, process isolation, situated studies, hierarchical task analysis, link analysis, operational diagram sequence, timeline analysis and GOMS has been analyzed to determine the learning outcomes. The learning outcomes have two categories: procedural and cognitive.

\section{Subsystem: learning outcomes}

The questioner module is a useful methodology to know the hypotheses generated by the users during the solution of the problem. Whit this kind of answers, the robot can estimate aspects such as student's beliefs, self-evaluation, biases, and heuristics used for reasoning, even the robot's effect [19].

As conclusion, the learning objectives are grouped into two categories. First, the objectives related to the knowledge of the problem. For example, the use of rules or operators to change states. Second, the objectives related to the knowledge of the strategy to solve the problem. For example, the recognition of the objectives to transform and reduce the problem, which is useful to implement the strategy of means-ends analysis.

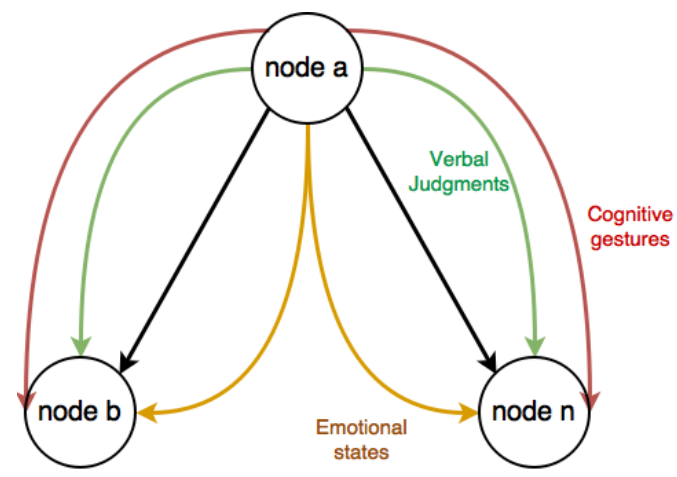

Figure 4: Graph characteristics

\section{SYSTEM III: SCAFFOLDING}

As mentioned above, Figure 1 shows the proposed artificial cognitive architecture in three modules. The third module plans and creates the intervention according to two conditions: learning curve (scaffolding) and Flow theory. After that, learning outcomes are prioritized and the intervention strategy is defined (See figure 1).

Figure 5 describes the Flow theory which is described as a balance between skills and task demands. The marron line represents the flow's evolution from a low level to a high level. The marron line divides the flow area into two equal parts. The axis $y$ represents the level of the challenge of the problem. For example, in the Hanoi tower, the number of the disks involved in the problem. The axis $x$ represents the user's skills. For example, the knowledge of the strategy. The $C i$ represents the current challenge level of the problem. The $S i$ represents the learner's skills which have been estimated by the previous subsystem. When the system determines the learner's flow level, three actions (c, e, s) are developed to bring closer the learner to the ideal flow zone.

\section{Subsystem: flow manager}

The flow manager is composed by four modules. First, learner's flow state. The learner's flow state acknowledges the balance between the learner's skills and the emotional state to change the cognitive load through different cognitive, emotional or physical actions. Second, support strategy selector. According to Figure 1, the robot has four strategies to support the learning process: focus lesson, guided instruction, collaborative work, and independent work. Third, role robot selector. The robot can take three different roles to support assertively the learning process: peer, tutor, and learner. Four, metacognitive trigger. The function is generated thinking process based on the graph evolution.

The learning process is foster through four strategies: focus lesson, guided instruction, cooperative work and independent work. By each strategy, a role is assigned. There are three alternatives: tutor, peer, and learner. As tutor, the robot interventions to explain and guide the learner's actions. As peer, the robot intervenes through verbal suggestions and physical interventions negotiated with the learner. As learner, the robot carry out questions related to the development of the task and through erroneous physical actions. In the three roles mentioned above, five aspects are implemented in robot: emotion, gestures, verbal judgments, kinematic description, positioning the blocks. 


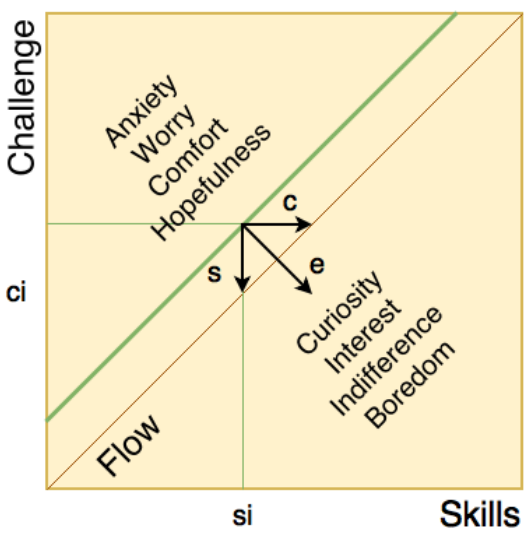

Figure 5: Flow Theory as decision robot's rule

The robot's morphology affects alternatives of gestural behavior and therefore its process of communication with the user. The robot can perform different kind of movements as emblematic, descriptive, rhythmic, deictic, symbolic, expressive and regulated. In humanoid robots, the non-verbal behavior of the robot affects the perception of the subjects [52]. In non-humanoid robots and without emotions: the behavior is related to the movement associated with the task.

\section{Subsystem: action manager}

According to the architecture and the scaffolding theory, robot decides which the best option to support the learning process. There are three ways. First, the robot gives emotions support. Second, the robot presents new information or gives support based on hits and missed done by the learner. The support's strategy could consider physical intervention as moving the blocks. Third, the robot changes the task's challenge. In this option, the robot changes the task's complexity and the number of blocks is reduced or augmented.

Emotional expression done by social robots during the learning process contribute to the feedback of task performance. Emotional manifestations expressed by the robot are: seeking information, attention, and interest, inviting and controlling interaction, influencing others and presenting their emotional state according to the conditions of a learning activity [50]. As a collaborative agent, robot has physical and cognitive artificial infrastructure (reason and emotion) to foster thought's actions during learning. One way is through emotional responses that allow increasing, refine and restructure the mental models of the learning situation. The way of expressing emotions, which is part of the personality of the robot, facilitates the understanding of the actions of the subject and affects his learning process.

The verbal judgments of the robot guide the student's learning process in two aspects: procedural and metacognitive. In the procedural aspect, verbal judgments made by the robot aim to guide the development of the problem posed using operators, recognition of rules and initial and target states. In the metacognitive aspect, the verbal judgments stimulate actions of thinking that guide the knowledge of the strategy of analysis of means-ends. Four actions proposed are to ask to evaluate the student's knowledge and understanding, to suggest facilitating the cognitive and meta-cognitive process, to point out to change the attention of the students, to explain in the moments where the student does not have enough knowledge of the problem or the implementation of the strategy to solve it [15].

As was mentioned above, the main mechanism of the scaffolding of the robot is the movement of the blocks to contribute to the process of solving the problem of transformation such as the Hanoi towers, the stair set and stacking blocks. The position of the blocks and the kinematic conditions for during positioning influence the user's thought processes [7].

In order to effectively support the robot, is necessary to consider aspects such as: adaptive behavior [39], use of skills learned from other tasks for use in new tasks [25], dynamic transition of responsibility between robot and learner [41], recognition of the cognitive state of the user [34], increase of dialogue resources through nonverbal behaviors [5], assertive suggestions appropriate to the needs of the learner [43][47][40][44].

In addition, support during decision-making requires consideration of aspects such as the dialogue system for the understanding of robot interventions [33][34], motor interaction requirements [23], The development of interactive behaviors through actions such as pointing objects [24], non-verbal actions such as gaze, proximity and development of iconic, metaphoric, deictic and vocal gestures robot [9].

The scaffolding process not only depends on student characteristics, also depends on the strategy and the task's characteristics. The above aspects open possibilities of generating levels of granularity in the scaffolding process. Figure 6 elicits a process of 
interaction with the robot. The physical appearance of the robot generates expectations of behavior, cognitive, emotional support, evaluation mechanisms and continuous feedback.

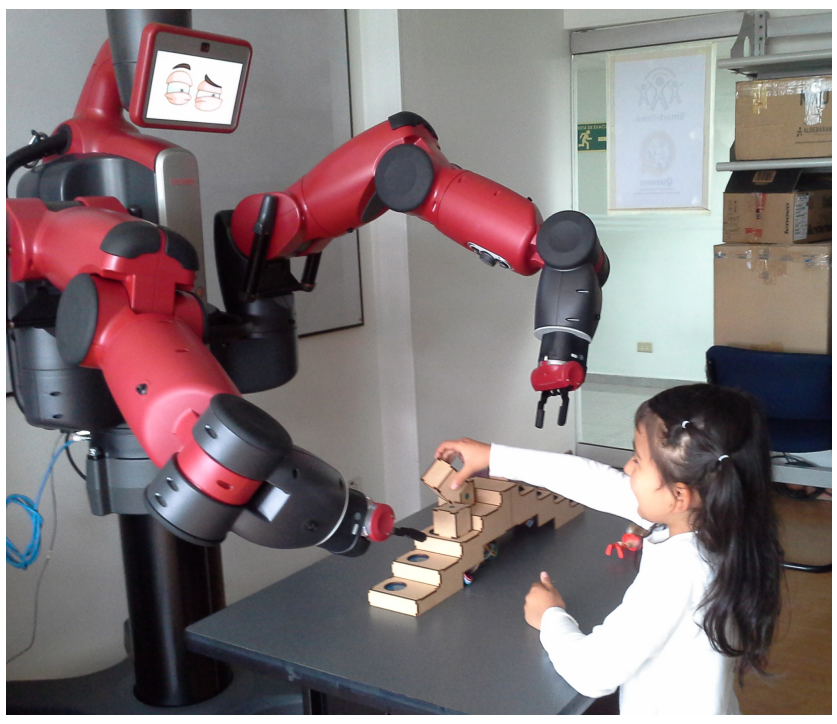

Figure 6: Baxter robot

\section{Discusion and Conclusions}

The technologies available these days are useful for countless applications. That is de case for robotics that can be adapted to different cases where the robot can be another participant in an environment created for a specific goal. That is the case of the present work where robots are used to establish Human-Robot interaction for the improvement of the learning environment. The same case could be used for the healthcare domain where tests are being designed for patients with mild dementia that are experiencing cognitive decline. Especially in older patients, the permanent availability of the robot without time constraints, can be useful as the older patients can use it when they are willing to make cognitive rehabilitation any time without the need of a therapist for those designed training exercises.

As conclusion, in education, robots have been considered as learning tools. They have evolved from simple tools that only followed students' instructions to complex cognitive artificial systems which allow robots to behave as tutor, peer, or learner. Each proposed behavior has been inspired by different pedagogical and psychological theories despite having been constrained by the contemporary technical conditions. Nowadays, embodiment, emotions, and physical interaction are topics of interest in the cognitive convergence challenge in the Human-Robot Interaction. For convergence to be effective and to contribute to the learning process, it is necessary for the cognitive architecture of the robot to develop three actions: to observe student behavior, to diagnose their cognitive and emotional states, and to intervene assertively according to their learning curve.

The robot as a collaborative agent recognizes several characteristics of the student and average during the learning process. The concept of mediation suggests an assertive intervention of the robot in a way that promotes cognitive effort during the development of the task and the student does not lose interest.

The shape of the robot influence the human-robot interaction and the behavior, which affects the user's understanding. In humanoid robots, the non-verbal behavior of the robot affects the perception of the subjects. In robots with the ability to manipulate and express emotions, the movement of the arms reflects the behavior. In non-humanoid robots and without emotions: the behavior is related to the movement associated with the task.

\section{ACKNOWLEDGMENTS}

\section{REFERENCES}

[1]. Alibali, M. W., Spencer, R. C., Knox, L., \& Kita, S. Spontaneous gestures influence strategy choices in problem solving. Psychological Science, 22(9), 1138-1144. 2011.

[2]. Alois Knoll, B Hildenbrandt, and Jianwei Zhang. Instructing cooperating assembly robots through situated dialogues in natural language. In Robotics and Automation, 1997. Proceedings., 1997 IEEE International Conference on, volume 1, pages 888-894. IEEE, 1997. 
[3]. Alois C Knoll. Distributed contract networks of sensor agents with adaptive reconfiguration: modelling, simulation, implementation and experiments. Journal of the Franklin Institute, 338(6):669-705, 2001.

[4]. A Knoll. A basic system for multimodal robot instruction. PRAGMATICS AND BEYOND NEW SERIES, pages 215-228, 2003.

[5]. Alves-Oliveira P., S. Janarthanam, A. Candeias, A. Deshmukh, T. Ribeiro, H. Hastie, A. Paiva, and R. Aylett. Towards dialogue dimensions for a robotic tutor in collaborative learning scenarios. Volume 2014-October, pages 862-867, 2014. doi: 10.1109/ROMAN.2014.6926361.

[6]. Bainbridge, W. A., Hart, J. W., Kim, E. S., \& Scassellati, B. (2011). The benefits of interactions with physically present robots over video-displayed agents. International Journal of Social Robotics, 3(1), 41-52.

[7]. Baxter, G. D., \& Ritter, F. E. (1996). Designing abstract visual perceptual and motor action capabilities for use by cognitive models. Technical Report 36. ERSC Center for Research and Development, Instruction and Training, Department of Pyschology, University of Nottingham, 1996.

[8]. Blauvelt, G. R., \& Eisenberg, M. (2006). Machineshop: a design environment for supporting children's construction of mechanical reasoning and spatial cognition. University of Colorado at Boulder, Boulder, CO.

[9]. Chandra S., P. Alves-Oliveira, S. Lemaignan, P. Sequeira, A. Paiva, and P. Dillenbourg. Can a child feel responsible for another in the presence of a robot in a collaborative learning activity volume 2015-November, pages 167-172, 2015. doi: 10.1109/ROMAN.2015.7333678.

[10]. Chrysafiadi, K., \& Virvou, M. (2015). Student Modeling for Personalized Education: A Review of the Literature. In Advances in Personalized Web-Based Education (pp. 1-24). Springer International Publishing.

[11]. Chu, M., \& Kita, S. (2011). The nature of gestures' beneficial role in spatial problem solving. Journal of Experimental Psychology: General, 140(1), 102.

[12]. Crandall, B., Klein, G. A., \& Hoffman, R. R. (2006). Working minds: A practitioner's guide to cognitive task analysis. Mit Press.

[13]. D Baker, R. S., Corbett, A. T., Roll, I., Koedinger, K. R., Aleven, V., Cocea, M., ... \& Mathews, M. (2013). Modeling and studying gaming the system with educational data mining. In International handbook of metacognition and learning technologies (pp. 97-115). Springer New York.

[14]. Essa, A. (2016). A possible future for next generation adaptive learning systems. Smart Learning Environments, 3(1), 16.

[15]. Fisher, D., \& Frey, N. (2010). Guided instruction: How to develop confident and successful learners. ASCD. Chicago.

[16]. Freire, T., Tavares, D., Silva, E., \& Teixeira, A. (2016). Flow, Leisure, and Positive Youth Development. In Flow Experience (pp. 163-178). Springer International Publishing.

[17]. Goldin-Meadow, S. (2006). Talking and thinking with our hands. Current Directions in Psychological Science, 15(1), 34-39.

[18]. Granados, L. F. M., \& Londoño, E. A. A. (2001). Análisis de Protocolos: Posibilidad metodológica para el estudio de procesos cognitivos. Universidad Pedagógica Nacional.

[19]. Hacker, D. J., Dunlosky, J., \& Graesser, A. C. (Eds.). (2009). Handbook of metacognition in education. Routledge.

[20]. Haibin Yan, Marcelo H Ang Jr, and Aun Neow Poo. A survey on perception methods for human-robot interaction in so- cial robots. International Journal of Social Robotics, 6(1):85-119, 2014.

[21]. Harmat, L., Andersen, F. Ø., Ullén, F., Wright, J., \& Sadlo, G. (Eds.). (2016). Flow Experience: Empirical Research and Applications. Springer.

[22]. Hayes, B., \& Scassellati, B. (2013). Challenges in shared-environment human-robot collaboration. Learning, Vol 8, page 9.

[23]. Jarrassé N., V. Sanguineti, and E. Burdet. Slaves no longer: Re- view on role assignment for human-robot joint motor ac- tion. Adaptive Behavior, 22(1):70-82, 2014. doi: 10.1177/ 1059712313481044. cited By 4

[24]. Kanda, T., Miyashita, T., Osada, T., Haikawa, Y., \& Ishiguro, H. (2008). Analysis of humanoid appearances in human-robot interaction. IEEE transactions on robotics, 24(3), 725-735.

[25]. Kelleher R Guerin, Sebastian D Riedel, Jonathan Bohren, and Gregory D Hager. Adjutant: A framework for flexible human-machine collaborative systems. In 2014 IEEE/RSJ International Conference on Intelligent Robots and Systems, pages 1392-1399. IEEE, 2014. 
[26]. Kim, M. C., \& Hannafin, M. J. (2011). Scaffolding problem solving in technology-enhanced learning environments (TELEs): Bridging research and theory with practice. Computers \& Education, 56(2), 403-417.

[27]. Kort, B., \& Reilly, R. (2002). Analytical models of emotions, learning and relationships: towards an affect-sensitive cognitive machine. In Conference on virtual worlds and simulation (VWSim 2002).

[28]. Kwak, S. S., Kim, Y., Kim, E., Shin, C., \& Cho, K. (2013, August). What makes people empathize with an emotional robot? The impact of agency and physical embodiment on human empathy for a robot. In RO-MAN, 2013 IEEE (pp. 180185). IEEE.

[29]. Mann, J. A., MacDonald, B. A., Kuo, I. H., Li, X., \& Broadbent, E. (2015). People respond better to robots than computer tablets delivering healthcare instructions. Computers in Human Behavior, 43, 112-117.

[30]. Manuel Giuliani, Mary Ellen Foster, Amy Isard, Colin Matheson, Jon Oberlander, and Alois Knoll. Situated reference in a hybrid human-robot interaction system. In Proceedings of the 6th International Natural Language Generation Conference, pages 67-75. Association for Computational Linguistics, 2010.

[31]. Manuel Giuliani and Alois Knoll. Using embodied multimodal fusion to perform supportive and instructive robot roles in human-robot interaction. International Journal of Social Robotics, 5(3):345-356, 2013.

[32]. Markus Rickert, Mary Ellen Foster, Manuel Giuliani, Tomas By, Giorgio Panin, and Alois Knoll. Integrating language, vision and action for human robot dialog systems. In Universal Access in Human-Computer Interaction. Ambient Interaction, pages 987-995. Springer, 2007.

[33]. Mary Ellen Foster, Ellen Gurman Bard, Markus Guhe, Robin L Hill, Jon Oberlander, and Alois Knoll. The roles of haptic- ostensive referring expressions in cooperative, task-based human-robot dialogue. In Proceedings of the 3rd ACM/IEEE international conference on Human robot interaction, pages 295-302. ACM, 2008a.

[34]. Mary Ellen Foster, Manuel Giuliani, Amy Isard, Colin Matheson, Jon Oberlander, and Alois Knoll. Evaluating description and reference strategies in a cooperative human-robot dialogue system. In IJCAI, pages 1818-1823, 2009.

[35]. Myrthe Tielman, Mark Neerincx, John-Jules Meyer, and Rose- marijn Looije. Adaptive emotional expression in robotchild interaction. In Proceedings of the 2014 ACM/IEEE international conference on Human-robot interaction, pages 407414. ACM, 2014.

[36]. National Research Council. (2005). Learning to think spatially: GIS as a support system in the K-12 curriculum. National Academies Press.

[37]. Neon B. Brooks, David Barner, Michael Frank, Susan Goldin-Meadow. (2015) The Role of Gesture in Supporting Mental Representations: The Case of Mental Abacus Arithmetic. University of Chicago.

[38]. Newell, A., \& Simon, H. A. (1972). Human problem solving (Vol. 104, No. 9). Englewood Cliffs, NJ: Prentice-Hall.

[39]. Pea, R. D. (2004). The social and technological dimensions of scaffolding and related theoretical concepts for learning, education, and human activity. The journal of the learning sciences, 13(3), 423-451.

[40]. Philippe Fournier-Viger, Roger Nkambou, Engelbert Mephu Nguifo, André Mayers, and Usef Faghihi. A multiparadigm intelligent tutoring system for robotic arm training. IEEE Transactions on Learning Technologies, 6(4):364377, 2013.

[41]. Ramacliandran A. and B. Scassellati. Adapting difficulty lev- els in personalized robot-child tutoring interactions. Volume WS-14-07, pages 56-59, 2014.

[42]. Reardon C., H. Zhang, R. Wright, and L.E. Parker. Response prompting for intelligent robot instruction of students with intellectual disabilities. Volume 2015-November, pages 784- 790, 2015. doi: 10.1109/ROMAN.2015.7333651.

[43]. Reardon, C., Zhang, H., Wright, R., \& Parker, L. E. (2015, August). Response prompting for intelligent robot instruction of students with intellectual disabilities. In Robot and Human Interactive Communication (RO-MAN), 2015 24th IEEE International Symposium on (pp. 784-790). IEEE.

[44]. Reidsma D., et. al. The easel project: Towards educational human- robot symbiotic interaction. Lecture Notes in Computer Science (including subseries Lecture Notes in Artificial Intelligence and Lecture Notes in Bioinformatics), 9793:297-306, 2016. doi: 10.1007/978- 3- 319-42417-0_27.

[45]. Serholt, C.A. Basedow, W. Barendregt, and M. Obaid. Com- paring a humanoid tutor to a human tutor delivering an in- structional task to children. Volume 2015-February, pages 1134-1141, 2015. doi: 10.1109/HUMANOIDS.2014.7041511.

[46]. Tabak, I. (2004). Synergy: A complement to emerging patterns of distributed scaffolding. The journal of the Learning Sciences, 13(3), 305-335. 
[47]. Takayuki Kanda, Takayuki Hirano, Daniel Eaton, and Hiroshi Ishiguro. Interactive robots as social partners and peer tutors for children: A field trial. Human-computer interaction, 19(1): 61-84, 2004.

[48]. Thien N.D., A. Terracina, L. Iocchi, and M. Mecella. Robotic teaching assistance for the "tower of hanoi" problem. Inter- national Journal of Distance Education Technologies, 14(1):64-76, 2016. doi: 10.4018/IJDET.2016010104.

[49]. Thomas Müller, Pujan Ziaie, and Alois Knoll. A wait-free real- time system for optimal distribution of vision tasks on multi- core architectures. In ICINCO-RA (1), pages 301-306, 2008.

[50]. Turner, J. E., Waugh, R. M., Summers, J. J., \& Grove, C. M. (2009). Implementing high-quality educational reform efforts: An interpersonal circumplex model bridging social and personal aspects of teachers' motivation. In Advances in teacher emotion research (pp. 253-271). Springer US.

[51]. Van De Sande, B. (2013). Properties of the Bayesian knowledge tracing model. JEDM-Journal of Educational Data Mining, 5(2), 1-10.

[52]. Maha Salem, Friederike Eyssel, Katharina Rohlfing, Stefan Kopp, and Frank Joublin. To err is human (-like): Effects of robot gesture on perceived anthropomorphism and likability. International Journal of Social Robotics, 5(3):313-323, 2013. 